\title{
The Effects of Moving Load on the Hydroelastic Response of a Very Large Floating Structure
}

\author{
Le Zhu $\mathbb{D},{ }^{1}$ Fei Shao $\mathbb{D},{ }^{1}$ Qian Xu $\mathbb{D}^{1},{ }^{1}$ Yonggang Sun, ${ }^{2}$ and Qingna $\mathrm{Ma}^{1}$ \\ ${ }^{1}$ College of Field Engineering, Army Engineering University of PLA, Nanjing 210007, China \\ ${ }^{2}$ Wuxi First Scientific Research Institute, Wuxi 214035, China \\ Correspondence should be addressed to Fei Shao; shaofei@seu.edu.cn and Qian Xu; 1058427910@qq.com
}

Received 27 August 2018; Accepted 13 November 2018; Published 3 December 2018

Academic Editor: Xiao-Qiao He

Copyright (c) 2018 Le Zhu et al. This is an open access article distributed under the Creative Commons Attribution License, which permits unrestricted use, distribution, and reproduction in any medium, provided the original work is properly cited.

\begin{abstract}
The hydroelastic response of a very large floating structure in regular waves suffering an external moving point load is considered. The linearized velocity potential theory is adopted to describe the fluid flow. To take into account the coupled effects of the structure deformation and fluid motion, the structure is divided into multiple segments and connected by an elastic beam. Then through adding a stiffness matrix arising from the elastic beam into the multiple bodies coupled motion equations, the hydroelastic response is considered. By applying the Fourier transform to the obtained frequency domain coefficients, the motion equation is transformed into the time domain and the external point load is further considered. The accuracy and effectiveness of the proposed method are verified through the comparison with experimental results. Finally, extensive results are provided, and the effects of the moving point load on the hydroelastic response of the very large floating structure are investigated in detail.
\end{abstract}

\section{Introduction}

Very large floating structure (VLFS) can be used as floating airports and bridges and for many other purposes. Due to the large dimension of the structure compared with waves, the elastic deformation should be considered when dealing with wave-structure interaction. Therefore, hydroelasticity method has been developed to account for coupling between hydrodynamic loading and structural deformation for floating flexible structures in waves.

Traditional hydroelasticity methods may be categorized into two different approaches, i.e., direct method and modesuperposition method. For the former, the equation of motion for a flexible structure is solved directly using conceptually full modes of the discretized system [1-3]. While for the latter, the hydroelastic response is obtained by dividing it into diffraction and radiation problems for each (flexible) mode of the flexible structure and then superposing all modal equations together to finally solve them. The modesuperposition method can be referred to Senjanović et al. [4] and Michailides et al. [5].

Different from the direct and mode-superposition methods, $\mathrm{Lu}$ et al. [6] proposed a frequency domain discrete-module-beam-bending based hydroelasticity method for a continuous flexible structure in waves. One advantage of this approach is that it avoids the need for predetermination of the flexible modes, which may be difficult for complicated geometric (or connection) features of the flexible structure. Sun et al. [7] extended the method proposed by Lu et al. [6] to be applicable for the hydroelastic response of a hinged VLFS in waves (in frequency domain). Other applications of this method can be referred to Xu et al. [8] and Zhang et al. [9].

For floating bridges and floating airport, the passage of vehicles or landing and take-off of an airplane will introduce unsteady external loading, which may affect the safety of the structure. Therefore, it is of practical importance to investigate at the design stage the transient hydrodynamic response of VLFSs. Traditionally, the hybrid frequency-time domain method based on mode-superposition approach was adopted by Kashiwagi $[10,11]$ to investigate the effects of unsteady external loading on VLFSs. Also the direct time domain method was used, for example, by Qiu [12] and Cheng et al. [13].

In the present study, the discrete-module-beam-bending based hydroelasticity method $[6,7,9]$ is used to investigate 


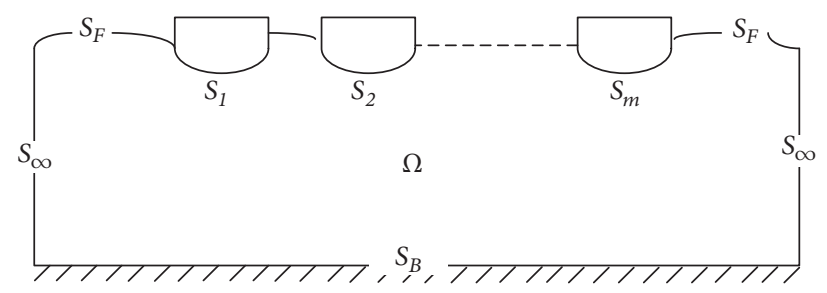

FIGURE 1: Definition of fluid and structure boundaries.

the hydroelastic response of a VLFS under unsteady external loading in waves. The effects of mass and moving velocity of the point loading on the hydroelastic response of the VLFS in waves are investigated in detail.

\section{Mathematical Model and Solution Procedure}

2.1. Governing Equation for the Multiple Floating Structures. To solve the problem, we assume that the fluid is inviscid, incompressible, and homogeneous, and the fluid flow is irrotational. Then the velocity potential $\Phi$ can be introduced to describe the fluid motion. Under the further assumption that the amplitude of the wave motion is small compared to its length and the dimension of the floating structure, we can apply the linearized velocity potential theory to describe the fluid flow.

We now consider the wave interactions between multiple floating structures, as shown in Figure 1. For sinusoidal fluid motion in time $t$ with radian frequency $\omega$, we may write the total velocity potential $\Phi$ into the following form:

$$
\Phi(x, y, z, t)=\operatorname{Re}\left(\phi e^{i \omega t}\right)
$$

with

$$
\begin{aligned}
\phi & =\varphi_{\mathrm{I}}+\varphi_{D}+\varphi_{R} \\
\text { and } \varphi_{R} & =\sum_{q=1}^{m} \varphi_{R, q}
\end{aligned}
$$

where $\operatorname{Re}$ indicates taking the real part, $\varphi_{\mathrm{I}}, \varphi_{D}$, and $\varphi_{R}$ are, respectively, the complex incident potential, diffracted potential, and the radiation potential. In fluid domain, the mass conservation requires that the velocity potential should satisfy the Laplace equation:

$$
\nabla^{2} \phi=0
$$

throughout the fluid domain $\Omega$. On the free surface $S_{F}$, the combined kinematic and dynamic boundary condition gives

$$
-\omega^{2} \phi+g \frac{\partial \phi}{\partial z}=0
$$

On the seabed $S_{B}$, the impermeable condition states

$$
\frac{\partial \phi}{\partial z}=0
$$

where the seabed is assumed to be flat. On the wetted structure surface $S_{q}(q=1, \ldots, m)$, the nonpenetration condition should be satisfied, i.e.,

$$
\frac{\partial \varphi_{D}}{\partial n}=-\frac{\partial \varphi_{I}}{\partial n}
$$

for the diffracted velocity potential, and

$$
\frac{\partial \varphi_{R, q}}{\partial n_{q}}=\vec{V}_{q} \cdot \vec{n}_{q}
$$

for the radiation velocity potential. Here, $q$ indicates the $q$-th floating structure. In the far field $S_{\infty}$, the radiation condition should be also enforced to close the problem,

$$
\begin{array}{r}
\lim _{R \rightarrow \infty} \sqrt{R}\left(\frac{\partial \phi_{D}}{\partial R}-i k \phi_{D}\right)=0 \\
\lim _{R \rightarrow \infty} \sqrt{R}\left(\frac{\partial \phi_{R, q}}{\partial R}-i k \phi_{R, q}\right)=0
\end{array}
$$

where $k$ denotes the wave number.

2.2. Solution Procedure for the Very Large Floating Structures. The boundary value problem described in (1) to (9) can be solved through the standard boundary element method. Here, the focus is put on the extension of the solution for multiple structure interaction problem to the hydroelastic response of very large floating structures. After the velocity potential has been solved, we can obtain the hydrodynamic pressure through the linearized Bernoulli equation.

In the frequency domain, the wave exciting force $\vec{F}_{\text {exk }}$ corresponding to the incident and diffracted velocity potentials can be given as

$$
\vec{F}_{\text {exk }}=i \omega \rho \iint_{S_{0 q}}\left(\varphi_{\mathrm{I}}+\varphi_{\mathrm{D}}\right) \cdot \vec{n}_{q} d S
$$

where $\rho$ is the density of water and $S_{0 q}$ represents the average wetted surface. The hydrodynamic force arising from the radiation potential can be expressed through the added mass $a_{k j}$ and damping coefficients $b_{k j}$, i.e.,

$$
\left[a_{k j}\right]+\frac{i}{\omega}\left[b_{k j}\right]=\rho \iint_{S_{0 q}} \varphi_{R, q} \cdot \vec{n}_{q} d S
$$

After the hydrodynamic forces are obtained, the equation of motion of freely floating multibody system can be given 
according to Newton second law. Here, it may be noticed that the multibody system comprises $m$ modules and each module has a six degree-of-freedom motion, as shown in Figure 1. For incident wave with unit amplitude, we have

$$
\begin{aligned}
& \left(-\omega^{2}\left(\left[M_{k}\right]+\left[a_{k k}\right]\right)-i \omega\left[b_{k k}\right]+\left[C_{k}\right]\right)\left\{u_{k}\right\} \\
& +\sum_{j=1, j \neq k}^{m}\left(-\omega^{2}\left[a_{k j}\right]-i \omega\left[b_{k j}\right]\right)\left\{u_{j}\right\}=\left\{f_{\text {exk }}\right\}
\end{aligned}
$$

for $k=1,2, \ldots, m$, where $\left[M_{k}\right]$ is the mass matrix of the $k$-th module, $\left[a_{k k}\right]$ is the added mass matrix of the $k$-th module, $\left[b_{k k}\right]$ is the damping coefficient matrix of the $k$-th module, $\left[C_{k}\right]$ is the hydrostatic stiffness matrix of the $k$-th module, $\left\{f_{\text {exk }}\right\}$ is the wave excitation force, and $\left\{u_{k}\right\}$ is the six degree motion amplitude of the $k$-th module and expressed as $\left\{u_{k x}, u_{k y}, u_{k z}, \alpha_{k}, \beta_{k}, \gamma_{k}\right\}^{T}$. The dimension is $6 \times 6$ for $\left[M_{k}\right]$, $\left[a_{k k}\right],\left[b_{k k}\right]$, and $\left[C_{k}\right]$ and $6 \times 1$ for $\left\{u_{k}\right\}$ and $\left\{f_{\text {exk }}\right\}(k, j=$ $1,2, \ldots, m)$. It should be noted that in (12), the constraint of displacement due to the existence of connections is not considered.

When considering the elastic response of the very large structure, (12) becomes

$$
\left(\begin{array}{cccc}
2 & {\left[a_{12}\right]} & \cdots & {\left[a_{1, n}\right]} \\
{\left[M_{1}\right]+\left[a_{11}\right]} & {\left[M_{2}\right]+\left[a_{22}\right]} & \cdots & {\left[a_{2, n}\right]} \\
\vdots & \vdots & \ddots & \vdots \\
{\left[a_{n, 1}\right]} & {\left[a_{n, 2}\right]} & \cdots & {\left[M_{n, n}\right]+\left[a_{n, n}\right]}
\end{array}\right]
$$

$$
-i \omega\left[\begin{array}{cccc}
{\left[b_{11}\right]} & {\left[b_{12}\right]} & \cdots & {\left[b_{1, n}\right]} \\
{\left[b_{21}\right]} & {\left[b_{22}\right]} & \cdots & {\left[b_{2, n}\right]} \\
\vdots & \vdots & \ddots & \vdots \\
{\left[b_{n, 1}\right]} & {\left[b_{n, 2}\right]} & \cdots & {\left[b_{n, n}\right]}
\end{array}\right]
$$

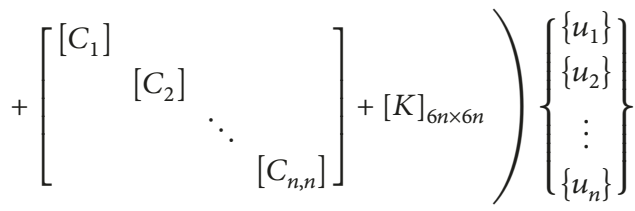$$
=\left\{\begin{array}{c}
\left\{f_{\text {ex1 }}\right\} \\
\left\{f_{\text {ex2 }}\right\} \\
\vdots \\
\left\{f_{\text {exn }}\right\}
\end{array}\right\}
$$

where $[K]$ is the term due to the connection between the submodules, and details can be seen in Sun et al. [7].

2.3. Motion Equation of Flexible Structure in Time Domain. To consider the effects of moving load on the hydroelastic response of the very large floating structure, the solution should be constructed in the time domain. To do this, in our work the hybrid frequency/time domain method (namely the time domain method based on impulse response function (IRF), which was first introduced by Cummins (1962)), is adopted to establish the motion equations. Here we consider a flexible structure in waves. The motion equations of the structure (which is divided into $m$ submodules) are established using the SAM approaches as follows:

$$
\begin{gathered}
{\left[\begin{array}{cccc}
{\left[M_{1}\right]+\left[a_{11}(\infty)\right]} & {\left[a_{12}(\infty)\right]} & \cdots & {\left[a_{1, n}(\infty)\right]} \\
{\left[a_{21}(\infty)\right]} & {\left[M_{2}\right]+\left[a_{22}(\infty)\right]} & \cdots & {\left[a_{2, n}(\infty)\right]} \\
\vdots & \vdots & \ddots & \vdots \\
{\left[a_{n, 1}(\infty)\right]} & {\left[a_{n, 2}(\infty)\right]} & \cdots & {\left[M_{n, n}\right]+\left[a_{n, n}(\infty)\right]}
\end{array}\right]\left\{\begin{array}{c}
\left\{\ddot{u}_{1}(t)\right\} \\
\left.\ddot{u}_{2}(t)\right\} \\
\vdots \\
\left.\ddot{u}_{n}(t)\right\}
\end{array}\right\}} \\
+\int_{0}^{t}\left[\begin{array}{cccc}
{\left[B_{21}(t-\tau)\right]} & {\left[B_{22}(t-\tau)\right]} & \cdots & {\left[B_{2, n}(t-\tau)\right]} \\
\vdots & \vdots & \ddots & \vdots \\
{\left[B_{n, 1}(t-\tau)\right]} & {\left[B_{n, 2}(t-\tau)\right]} & \cdots & {\left[B_{n, n}(t-\tau)\right]}
\end{array}\right]\left\{\begin{array}{c}
\left\{\dot{u}_{1}(t)\right\} \\
\vdots \\
\left\{\dot{u}_{2}(t)\right\} \\
\vdots
\end{array}\right\} d \tau+\left[K_{\text {Total }}\right]_{6 n \times 6 n}\left\{\begin{array}{c}
\left\{u_{1}\right\} \\
\left\{u_{2}\right\} \\
\vdots \\
\left\{u_{n}\right\}
\end{array}\right\}\left\{\begin{array}{c}
\left\{f_{\text {ex1 }}\right\} \\
\left\{f_{\text {ex }}\right\} \\
\vdots \\
\left\{f_{\text {exnn }}\right\}
\end{array}\right\}
\end{gathered}
$$

where $\left[a_{i j}(\infty)\right]$ is the added mass matrix of the $i$-th module caused by the motion of the $j$-th module at frequency of infinity. $\left[B_{i j}(t)\right]$ is the kernel function of the $i$-th module caused by the motion of the $j$-th module, which is related to the radiation damping matrix $\left[b_{i j}(\omega)\right]$. The expressions of $\left[a_{i j}(\infty)\right]$ and $\left[B_{i j}(t)\right]$ are given as follows:

$$
\begin{aligned}
{\left[a_{i j}(\infty)\right]=[} & \left.a_{i j}\left(\omega_{a c}\right)\right] \\
& +\frac{1}{\omega_{a c}} \int_{0}^{\infty}\left[B_{i j}(t)\right] \mathrm{s} \in\left(\omega_{a c} t\right) d t
\end{aligned}
$$

$$
\left[B_{i j}(t)\right]=\frac{2}{\pi} \int_{0}^{\infty}\left[b_{i j}(\omega)\right] \cos (\omega t) d \omega
$$

where $\omega_{a c}$ is an arbitrarily chosen frequency.

The total stiffness matrix $\left[K_{\text {Total }}\right]$ is composed of two parts, i.e., the hydrostatic stiffness of the structure [C] and the structural stiffness matrix (considering the deformation) $[K]$. The expression of $\left[K_{\text {Total }}\right]$ is given as follows:

$$
\left[K_{\text {Total }}\right]_{6 n \times 6 n}=[C]+[K]
$$




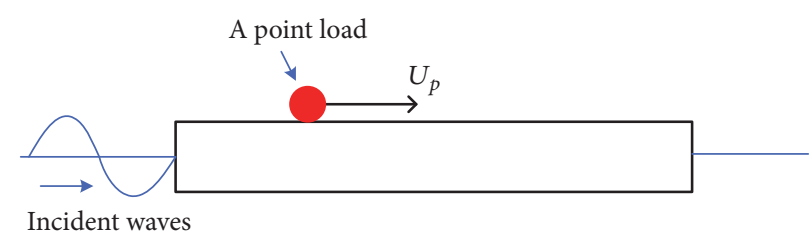

FIgURE 2: A schematic of a flexible structure with moving point load on the upper surface in waves.

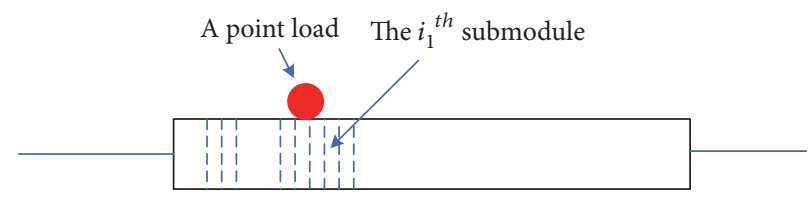

FIGURE 3: A schematic of a static point load acting on a flexible structure in calm water.

$$
\begin{aligned}
&=\left[\begin{array}{ccccc}
{\left[C_{1}\right]} & & & & \\
& \ddots & & & \\
& & {\left[C_{k}\right]} & & \\
& & & \ddots & \\
& & & & {\left[C_{n}\right]}
\end{array}\right] \\
&+[K]_{6 n \times 6 n}
\end{aligned}
$$

2.4. Consideration of a Moving Load. For a floating bridge or airport, there are vehicles moving on the upper surface of the structure. Usually the size of vehicles is small compared with the floating structure, which means that the vehicle can be regarded as a point load. Thus the problem of a vehicle moving on the floating bridge (or airport) can be simplified as a point load moving on the upper surface of a flexible structure. In this section, the time domain method is adopted to investigate the dynamic response of a flexible structure with moving point loads on the upper surface in waves (see Figure 2).

The linear assumption for the wave-structure interaction still remains here. Besides, we assume that the existence of the moving point load does not cause large structural deformation. Thus the floating system shown in Figure 2 is still a linear system. The point mass is assumed to move in a uniform horizontal velocity $U_{p}$. The force due to the point load on the structure is denoted as $\left\{\bar{f}_{p}(t)\right\}_{6 \times 1}$. As the motion equation is established on the center of each submodule of the structure. Thus the force $\left\{\bar{f}_{p}(t)\right\}$ should be transformed to the ones on the center of submodules, which is denoted as $\left\{f_{p}(t)\right\}_{6 n \times 1}=\left(\left\{f_{p 1}(t)\right\} \quad \ldots \quad\left\{f_{p n}(t)\right\}\right)^{T}$.

In order to obtain the relationship between $\left\{\bar{f}_{p}(t)\right\}$ and $\left\{f_{p}(t)\right\}$, we first consider a (static) point load acting on a flexible structures in calm water. The motion equation (can be obtained from (14) after some manipulation) is given as follows:

$$
\left[K_{\text {Total }}\right]_{6 n_{1} \times 6 n_{1}}\left\{\begin{array}{c}
\left\{u_{1}\right\} \\
\left\{u_{2}\right\} \\
\vdots \\
\left\{u_{n_{1}}\right\}
\end{array}\right\}=\left\{\begin{array}{c}
\left\{f_{p 1}\right\} \\
\left\{f_{p 2}\right\} \\
\vdots \\
\left\{f_{p n_{1}}\right\}
\end{array}\right\}
$$

It should be noted that in calm water, the whole structure is divided into $m_{1}$ submodules. $m_{1}$ is a large number $\left(m_{1}=\right.$ 150 adopted in this section) and is much larger than the number of submodule used in hydroelastic response, $m(m=$ 8 in the present analysis). The reason is that by setting a series of submodules (or elements) of very small size, the point load can be regarded as acting on the center of one particular submodule (for example, the $i_{1}^{\text {th }}$ submodule shown in Figure 3). Then the equivalent force satisfies $\left\{f_{p j}\right\}=0$ $\left(j \neq i_{1}\right)$ and $\left\{f_{p j}\right\}=\left\{\bar{f}_{p}(t)\right\}\left(j=i_{1}\right)$.

By solving (18), we obtain the distribution of displacement along the structure in calm water caused by the static point load at a given position on the structure. Then the displacement at the center of submodules (the number is $m),\{u\}_{6 m \times 1}$ for hydroelastic calculation (see (14)), is also obtained. Thus we can obtain the equivalent force $\left\{f_{p}\right\}_{6 m \times 1}$ as follows:

$$
\left\{\begin{array}{c}
\left\{f_{p 1}\right\} \\
\left\{f_{p 2}\right\} \\
\vdots \\
\left\{f_{m}\right\}
\end{array}\right\}=\left[K_{\text {Total }}\right]_{6 m \times 6 m}\left\{\begin{array}{c}
\left\{u_{1}\right\} \\
\left\{u_{2}\right\} \\
\vdots \\
\left\{u_{m}\right\}
\end{array}\right\}
$$

In calm water, we can obtain the equivalent force $\left\{f_{p}\right\}_{6 m \times 1}$ for static point loads acting on any position of the structure. Finally, the hydroelastic response equation can be written as 


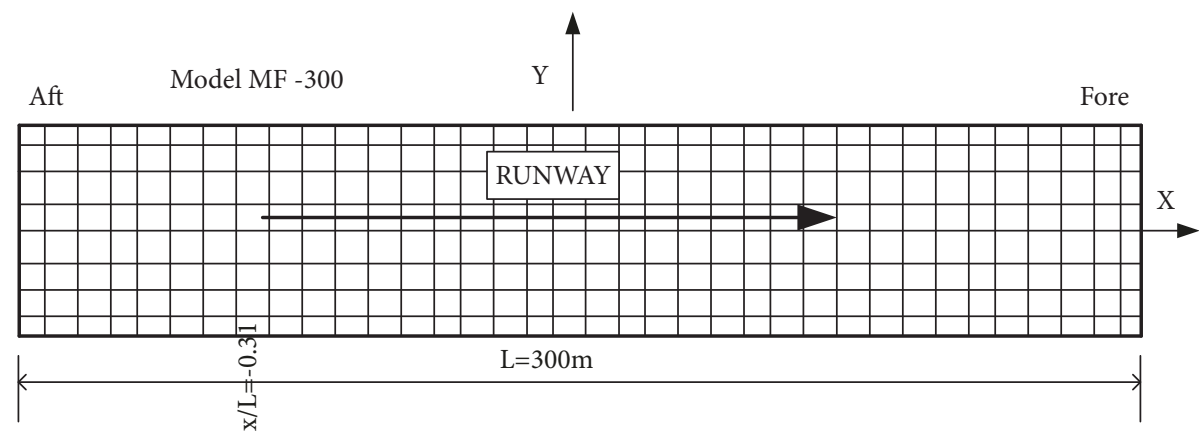

Figure 4: Plan view of the flexible VLFS prototype.

$$
\begin{aligned}
& {\left[\begin{array}{cccc}
{\left[M_{1}\right]+\left[a_{11}(\infty)\right]} & {\left[a_{12}(\infty)\right]} & \cdots & {\left[a_{1, n}(\infty)\right]} \\
{\left[a_{21}(\infty)\right]} & {\left[M_{2}\right]+\left[a_{22}(\infty)\right]} & \cdots & {\left[a_{2, n}(\infty)\right]} \\
\vdots & \vdots & \ddots & \vdots \\
{\left[a_{n, 1}(\infty)\right]} & {\left[a_{n, 2}(\infty)\right]} & \cdots & {\left[M_{n, n}\right]+\left[a_{n, n}(\infty)\right]}
\end{array}\right]\left\{\begin{array}{c}
\left\{\ddot{u}_{1}(t)\right\} \\
\left\{\ddot{u}_{2}(t)\right\} \\
\vdots \\
\left\{\ddot{u}_{n}(t)\right\}
\end{array}\right\}} \\
& +\int_{0}^{t}\left[\begin{array}{cccc}
{\left[B_{11}(t-\tau)\right]} & {\left[B_{12}(t-\tau)\right]} & \cdots & {\left[B_{1, n}(t-\tau)\right]} \\
{\left[B_{21}(t-\tau)\right]} & {\left[B_{22}(t-\tau)\right]} & \cdots & {\left[B_{2, n}(t-\tau)\right]} \\
\vdots & \vdots & \ddots & \vdots \\
{\left[B_{n, 1}(t-\tau)\right]} & {\left[B_{n, 2}(t-\tau)\right]} & \cdots & {\left[B_{n, n}(t-\tau)\right]}
\end{array}\right]\left\{\begin{array}{c}
\left\{\dot{u}_{1}(t)\right\} \\
\left\{\dot{u}_{2}(t)\right\} \\
\vdots \\
\left\{\dot{u}_{n}(t)\right\}
\end{array}\right\} d \tau+\left[K_{\text {Total }}\right]_{6 n \times 6 n}\left\{\begin{array}{c}
\left\{u_{1}\right\} \\
\left\{u_{2}\right\} \\
\vdots \\
\left\{u_{n}\right\}
\end{array}\right\}=\left\{\begin{array}{c}
\left\{f_{\text {ex } 1}\right\} \\
\left\{f_{\text {ex } 2}\right\} \\
\vdots \\
\left\{f_{\text {exn }}\right\}
\end{array}\right\}
\end{aligned}
$$

$$
+\left\{\begin{array}{c}
\left\{f_{p 1}\right\} \\
\left\{f_{p 2}\right\} \\
\vdots \\
\left\{f_{p n}\right\}
\end{array}\right\}
$$

with the external load considered.

\section{Numerical Results}

3.1. Validation of the Method. To verify the accuracy and efficiency of the method presented in this paper, we consider the model by Yago and Endo (1996), which is a scaled model of the Mega-Float. This is constructed and developed for use in sheltered waters. The wave amplitude is $1 m$, and the main parameters are listed in Table 1 . The number of nodes in hydrodynamic calculation of VLFS is $160 \times 10 \times 10$.

The case considered here is the moving load experiment, conducted by Endo and Yago (1999). The experiment model VL-10 was designed similar to the continuous flexible VLFS model adopted by Yago and Endo (1996) on the basis of similitude (with a scaling factor of 30.77). In the following test, all physical properties are listed in prototype scale (i.e., the scale of MF-300). In experiment, a moving load of $201016 \mathrm{~kg}$ was navigated on the runway from $x / L=$ $-(0.31 \sim 0.4)$ (see Figure 4 ) with constant velocity $3.39 \mathrm{~m} / \mathrm{s}$.
It should be noted that the origin is set in the middle of the structure in Endo and Yago (1999), which is different from the definition of origin at left side of the structure in previous sections in the present study. This case is also calculated by the present numerical method. As no incident waves are considered, the wave excitation force in the right hand side of (12) should be ignored. The time histories of vertical displacement of the structure obtained from the present numerical method, "Present", and from the experiment, "Experiment", are comparatively shown in Figure 5. It can be seen that the calculated results are found to show a reasonable agreement with the experiment, which validates the proposed numerical methodologies for calculating the dynamic response of a flexible structure undergoing moving point loads. Generally, it can be expected that the vertical displacement of the VLFS will have a large trough near the moving load. This means that the vertical displacement at $x / L=-0.25,0$ and 0.5 will have a large trough when $t \approx 5.3 s$, $27.4 s$, and $71.7 s$, respectively, as shown in Figure 5. For the black line of $x / L=0.5$, since the large trough corresponding 


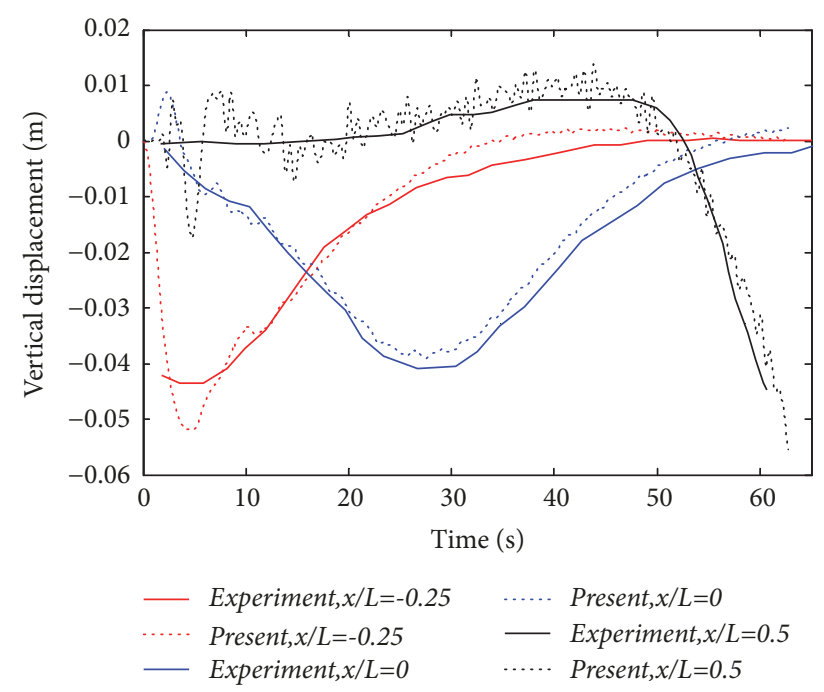

Figure 5: Time history of vertical displacement of the flexible structure in calm water.

TABle 1: Parameters of the model.

\begin{tabular}{lcc}
\hline Parameters & Units & Value \\
\hline Length & L: $\mathrm{m}$ & 300 \\
Width & B: $\mathrm{m}$ & 60 \\
Depth & $\mathrm{D}: \mathrm{m}$ & 2 \\
Draft & $\mathrm{d}: \mathrm{m}$ & 0.5 \\
Young modulus & $\mathrm{E}: \mathrm{N} / \mathrm{m}^{2}$ & $1.19 \mathrm{e} 10$ \\
Poisson ratio & $\nu$ & 0.13 \\
Density & $\rho: \mathrm{kg} / \mathrm{m}^{3}$ & 256.25 \\
Water depth & $\mathrm{H}: \mathrm{m}$ & 58.5 \\
\hline
\end{tabular}

to $t=71.7 \mathrm{~s}$ is out of the computed time range, thus only a trend to the large trough is observed in the figure.

3.2. The Effects of Moving Load Mass. We then consider the effects of the mass of the moving point load on the hydroelastic response of the very large floating structure. The incident wave length is taken to be $300 \mathrm{~m}$, and the incident wave angle is chosen as zero or heading waves. Three different moving point masses are considered, i.e., $200 t, 400 t, 600 t$, and $800 t$. For each case, the mass point load is moving with the same velocity or $5 \mathrm{~m} / \mathrm{s}$.

In Figure 6, we show the typical contour plot of the vertical displacement and bending moment response of the VLFS in regular wave with a moving point mass $600 t$, against time and longitudinal position of the structure. In both Figures 6(a) and 6(b), the trajectory of the moving point load is also given. It can be seen from Figure 6(a) that the absolute value of the displacement at the two end sides of the VLFS is usually larger than those for the point within the VFLS. This is not unexpected because due to that both the two end sides are free to move. From the figure, we can also see that the largest vertical displacement happens when the moving point load is located at the two end sides of the VLFS, i.e., approximately at $40 \mathrm{~s}$ and $100 \mathrm{~s}$, and the value is always

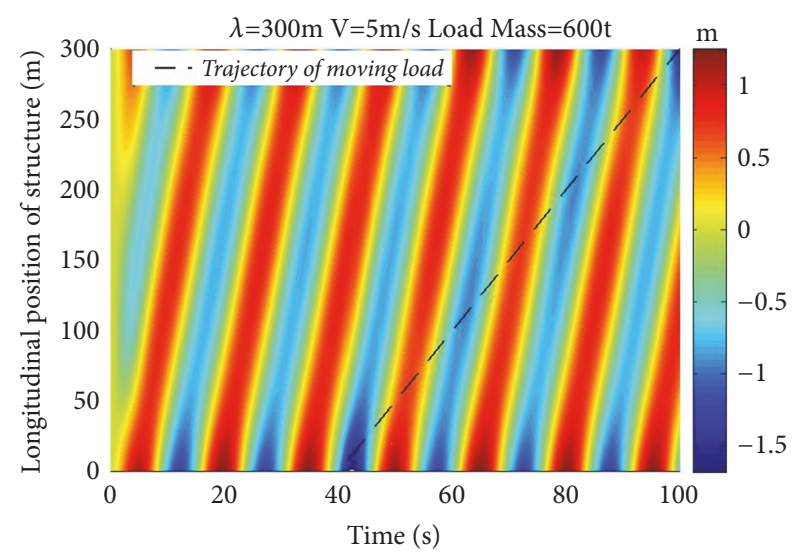

(a) Vertical displacement response of the VLFS

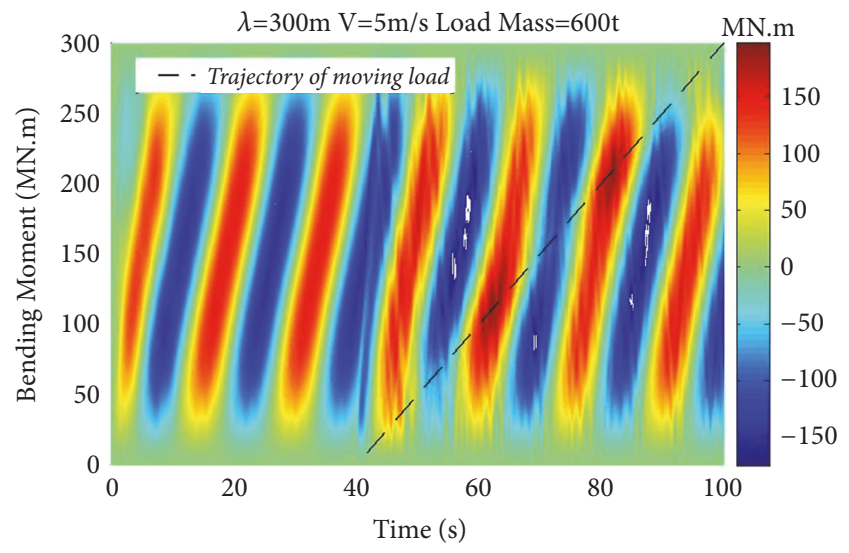

(b) Bending moment response of the VLFS

FIgURE 6: Typical contours of the vertical displacement and bending moment responses of the VLFS in regular wave with a moving point mass as $600 t$.

negative. This is because the moving point load is always negative due to the gravity. Compared with Figure 6(a), it can be seen from Figure 6(b) that the bending moment response of the VLFS around the middle point is always larger than those at the two end sides of the VLFS, which is coincided with the free beam theory. Especially, from these two figures we can see that the effects of the moving point load on the bending moment response of the VLFS are much larger than those on the vertical displacement, as shown for the region $(40 s, 100 s)$.

Computations are then carried out for the vertical displacement and bending moment responses of the VLFS in regular waves undergoing a moving point load with different masses, as shown in Figures 7(a) and 7(b), respectively, for the vertical displacement response and bending moment response. From Figure 7(a) we can see that with the increase of the moving point load mass, the absolute values of the peaks become much smaller while the absolute values of the troughs become much larger. This is for that the overall effect of the moving load is inducing a negative displacement. In addition, since the time histories are for the point located at $x / L=0.5$, thus within $(60 s, 80 s)$ the effects of the moving 


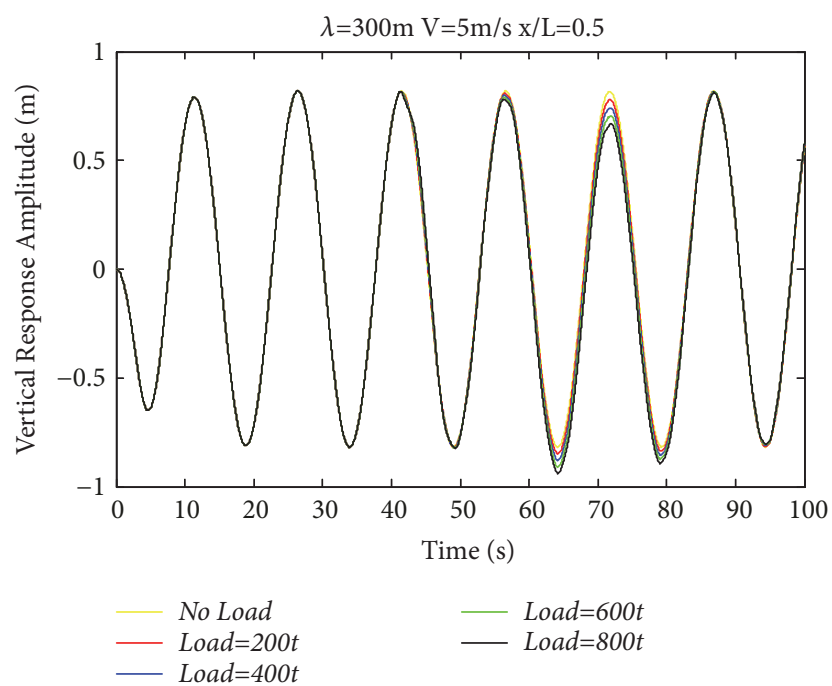

(a) Vertical displacement response of the VLFS

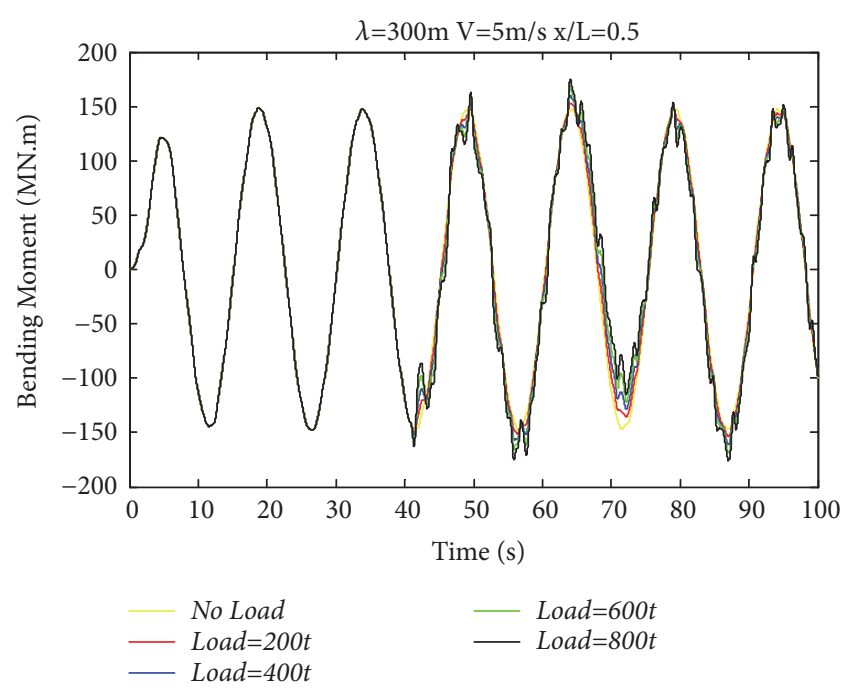

(b) Bending moment response of the VLFS

FIGURE 7: Time histories of the vertical displacement and bending moment responses of the VLFS in regular wave for a moving point load with different masses.

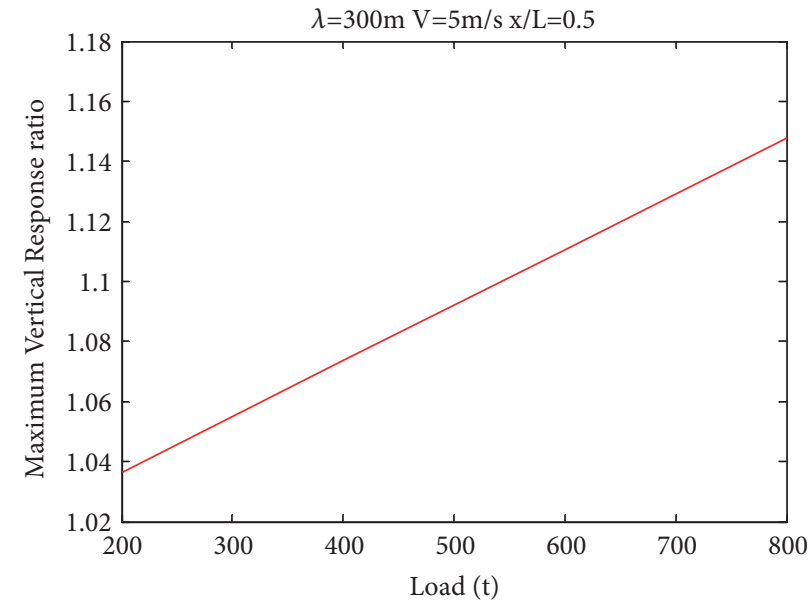

(a) Vertical displacement response of the VLFS

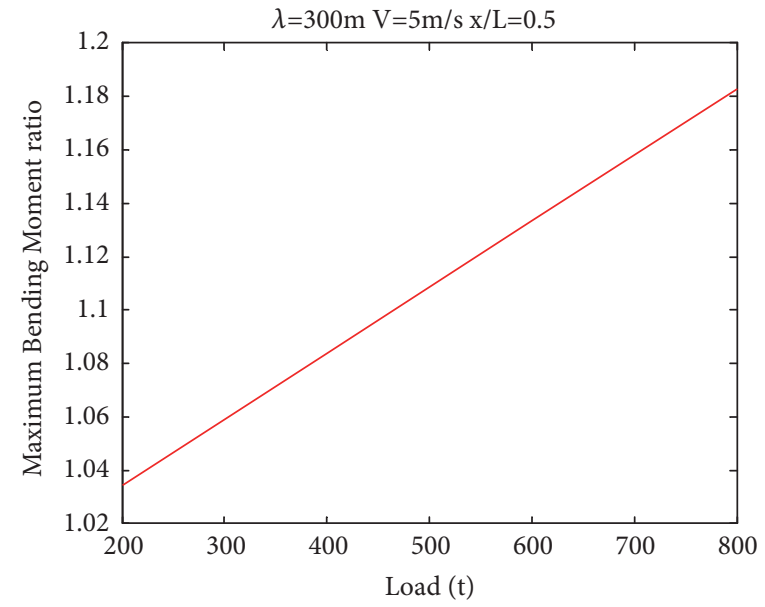

(b) Bending moment response of the VLFS

FIGURE 8: The maximum vertical displacement and bending moment responses of the VLFS in regular wave for a moving point load with different masses.

load are most obvious. From Figure 7(b) it can be seen that the absolute values of the peaks and troughs for the case with a moving load are always larger than those without a moving load. Compared with Figure 7(a), we can see that once the moving load is on the VLFS, its effects should be always considered. In addition, it is interesting to see that compared with the vertical displacement response, which varies with a single frequency, there are more frequency components in the bending moment response, which indicates that the hydroelastic effects must be taken into account to obtain a reasonable result. To see the effects of the moving load mass more clearly, in Figures 8(a) and 8(b) we plot the maximum vertical displacement and bending moment against the load mass, both of which increase with load mass linearly.
3.3. The Effects of Moving Load Velocity. Finally, we investigate the effects of the moving load velocity on the vertical displacement and bending moment response of the VLFS. The incident wave length and wave angle are taken to be $300 \mathrm{~m}$ and zero degree, respectively. The moving point mass is chosen as 600t. Here, four moving velocities are considered, i.e., $5 \mathrm{~m} / \mathrm{s}, 10 \mathrm{~m} / \mathrm{s}, 15 \mathrm{~m} / \mathrm{s}$, and $20 \mathrm{~m} / \mathrm{s}$. In Figures 9 (a) and 9 (b) we show the typical contour plots of the vertical displacement and bending moment response, respectively, in which the moving point load velocity is $15 \mathrm{~m} / \mathrm{s}$. The comparison of Figures 9(a) and 9(b) shows that when the moving point load leaves the VLFS, its effects on the vertical displacement disappear quickly, while its effects on the bending moment response will maintain for a long time. Also, from the comparison of Figure 9(b) with Figure 6(b) we can see that 


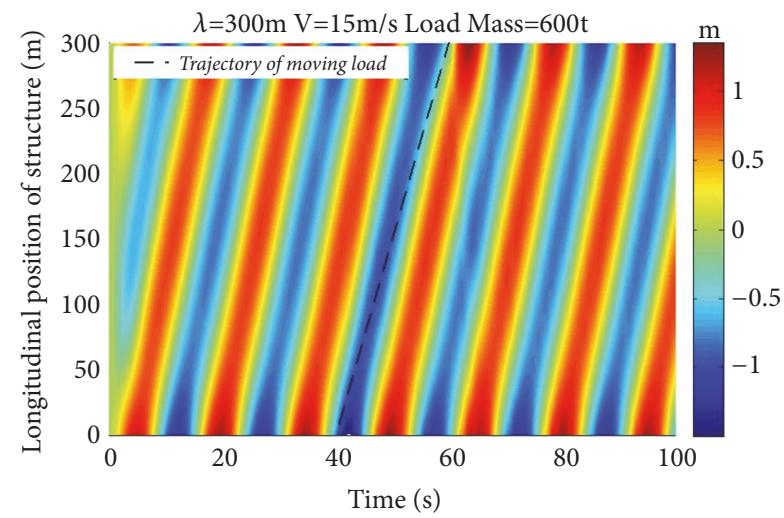

(a) Vertical displacement response of the VLFS

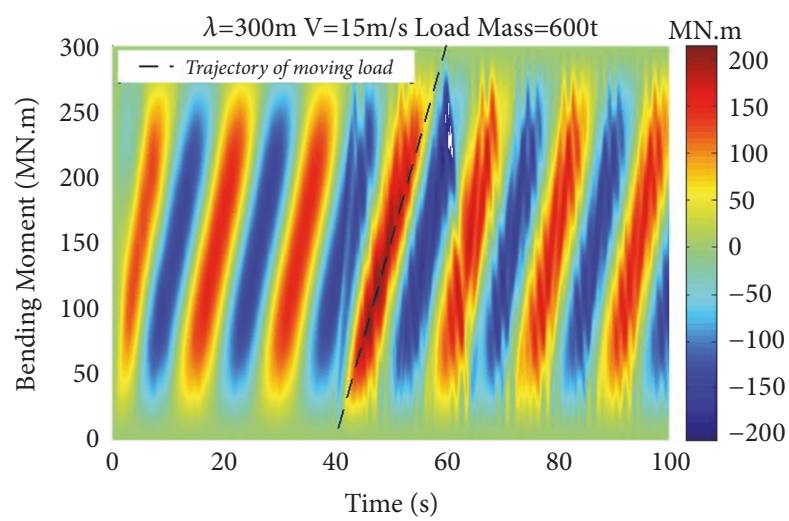

(b) Bending moment response of the VLFS

FIGURE 9: Typical contours of the vertical displacement and bending moment responses of the VLFS in regular wave for a moving point mass with velocity as $15 \mathrm{~m} / \mathrm{s}$.

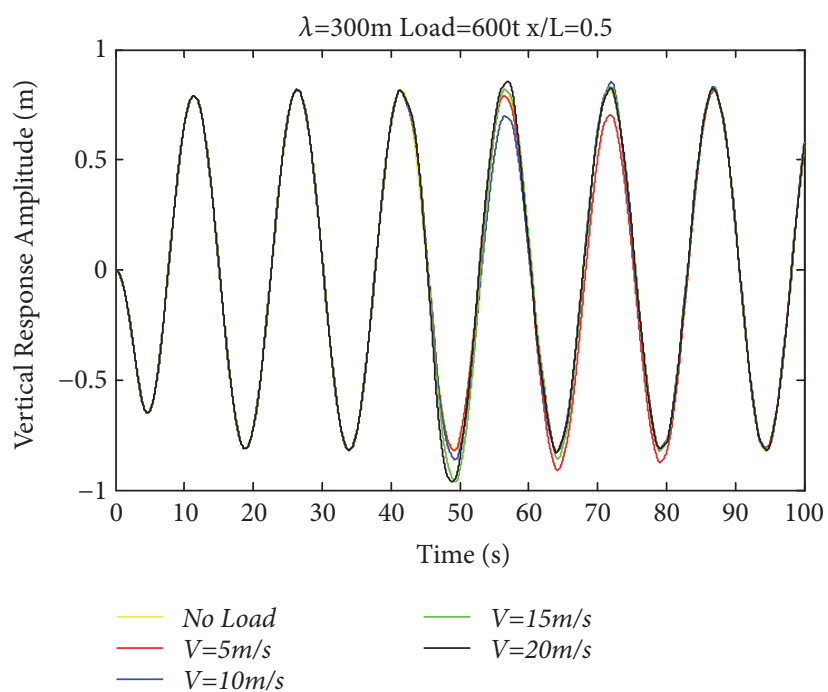

(a) Vertical displacement response of the VLFS

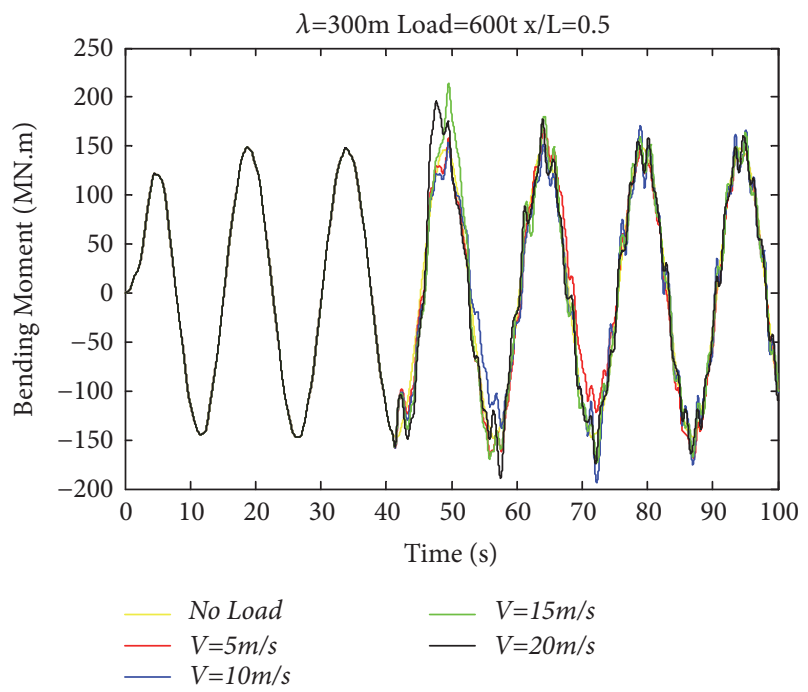

(b) Bending moment response of the VLFS

FIGURE 10: Time histories of the vertical displacement and bending moment responses of the VLFS in regular wave for a moving point load with different velocities.

the effects of the moving point load on the bending moment response are much more obvious for a larger moving velocity.

In Figures 10(a) and 10(b) we show the time histories of the vertical displacement and bending moment response of the VLFS in regular waves undergoing a moving point load with four different velocities. The point load moves on the VLFS at $40 s$ and leaves the VLFS at $60 s$. The results for the case without the moving point mass are also given. From this figure it can be observed that for each velocity, after the point load leaves the VLFS, its effects will maintain a few times, and then disappear. Within the computational range of moving velocity, there is no evidence to show that the vertical displacement or the bending moment increase or decrease with the moving velocity. However, the overall trend is that the vertical displacement and bending moment response for VLFS with a moving point load are generally larger than those without the moving point load. This can be more clearly seen in Figures 11(a) and 11(b), which shows the maximum vertical displacement and bending moment response of the VLFS against point load velocity.

\section{Conclusions}

In this paper, we have presented the solution procedure for the hydroelastic response of a very large floating structure in regular waves suffering an external moving point load, based on the linearized velocity potential theory and the multiple bodies beam method. The solution starts from the coupled motion equations for multiple freely floating bodies. Then assuming these bodies are connected through an elastic beam or adding an additional stiffness matrix into the coupled motion equations, the coupled effects of the structure deformation and fluid motion are considered. Through further transforming the frequency domain motion 


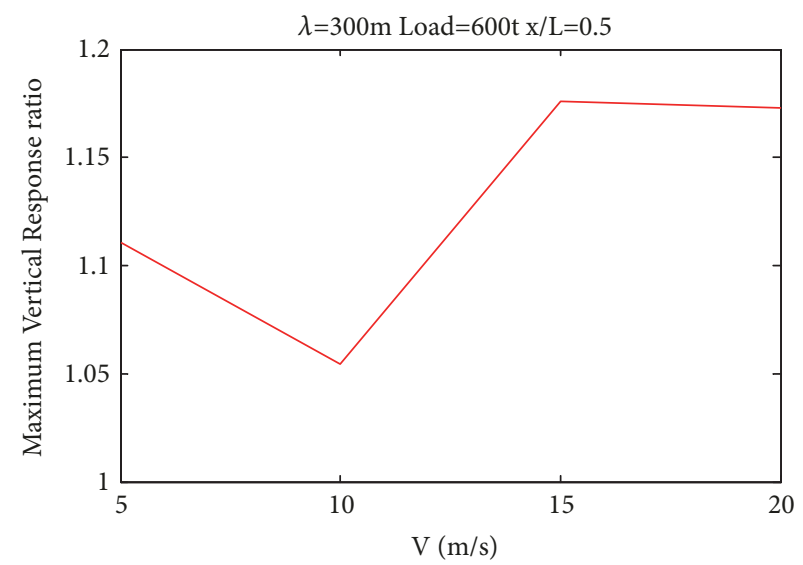

(a) Vertical displacement response of the VLFS

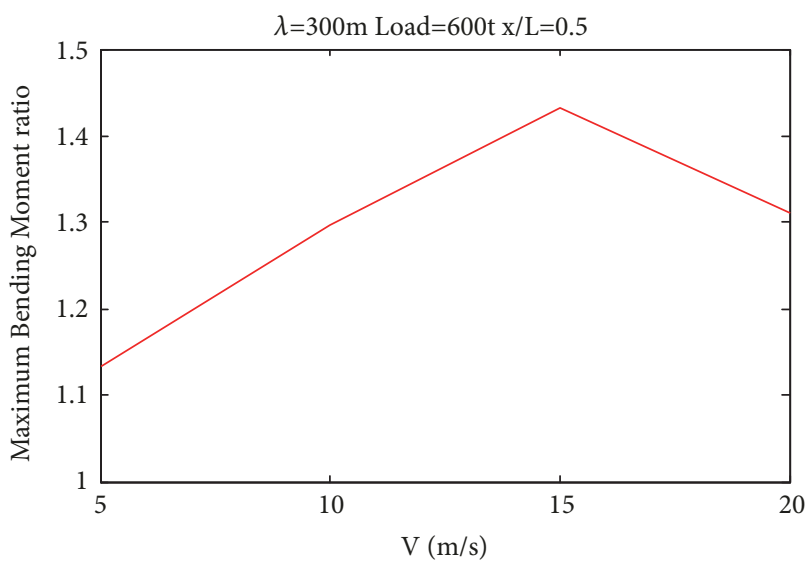

(b) Bending moment response of the VLFS

FIGURE 11: The maximum vertical displacement and bending moment responses of the VLFS in regular wave for a moving point load with different velocities.

equations into the time domain ones via Fourier transform, the effects of the moving point load are taken into account. The comparison with experiment results shows that the method is accurate enough for engineering application. The extensive results indicate that both the vertical displacement and bending moment response will increase with the moving point load mass. However, there is no evidence to show that the vertical displacement or the bending moment increases or decreases with the moving velocity.

\section{Data Availability}

All data used to support the findings of this study are provided in full in the Results section of this paper, which are shown in form of curves and contours.

\section{Conflicts of Interest}

The authors declare that there are no conflicts of interest regarding the publication of this paper.

\section{Acknowledgments}

The authors would like to highly appreciate Dr. Zhifu Li from Jiangsu University of Science and Technology for his zealous and generous discussions. This work is funded by project (2014CB046801) supported by the National Basic Research Development Program of China (973 Program), project (51208261) supported by the National Science Foundation of China, and project (2016M602972) supported by the Postdoctoral Science Foundation of China.

\section{References}

[1] R. Eatock Taylor, "Wet or dry modes in linear hydroelasticitywhy modes," in Hydroelasticity in marine technology, pp. 239250, Oxford, UK, 2003.

[2] R. Eatock Taylor, "Hydroelastic analysis of plates and some approximations," Journal of Engineering Mathematics, vol. 58, no. 1-4, pp. 267-278, 2007.
[3] B. W. Kim, S. Young Hong, J. H. Kyoung, and S. Kyu Cho, "Evaluation of bending moments and shear forces at unit connections of very large floating structures using hydroelastic and rigid body analyses," Ocean Engineering, vol. 34, no. 11-12, pp. 1668-1679, 2007.

[4] I. Senjanović, Š. Malenica, and S. Tomašević, "Investigation of ship hydroelasticity, Ocean Engineering, vol. 35, no. 5-6, pp. 523-535, 2008

[5] C. Michailides, E. Loukogeorgaki, and D. C. Angelides, "Response analysis and optimum configuration of a modular floating structure with flexible connectors," Applied Ocean Research, vol. 43, pp. 112-130, 2013.

[6] D. Lu, S. Fu, X. Zhang, F. Guo, and Y. Gao, "A method to estimate the hydroelastic behaviour of VLFS based on multi-rigid-body dynamics and beam bending," Ships and Offshore Structures, pp. $1-9,2016$

[7] Y. Sun, D. Lu, J. Xu, and X. Zhang, "A study of hydroelastic behavior of hinged VLFS," International Journal of Naval Architecture and Ocean Engineering, vol. 10, no. 2, pp. 170-179, 2018.

[8] J. Xu, Y. Sun, Z. Li, X. Zhang, and D. Lu, "Analysis of the Hydroelastic Performance of Very Large Floating Structures Based on Multimodules Beam Theory," Mathematical Problems in Engineering, vol. 2017, Article ID 6482527, 14 pages, 2017.

[9] X. Zhang, D. Lu, Y. Gao, and L. Chen, "A time domain discrete-module-beam-bending-based hydroelasticity method for the transient response of very large floating structures under unsteady external loads," Ocean Engineering, vol. 164, pp. 332349, 2018.

[10] M. Kashiwagi, "A time-domain mode-expansion method for calculating transient elastic responses of a pontoon-type VLFS," Journal of Marine Science and Technology, vol. 5, no. 2, pp. 89$100,2000$.

[11] M. Kashiwagi, “Transient responses of a VLFS during landing and take off of an airplane," Journal of Marine Science and Technology, vol. 9, no. 1, pp. 14-23, 2004.

[12] L.-C. Qiu, "Numerical simulation of transient hydroelastic response of a floating beam induced by landing loads," Applied Ocean Research, vol. 29, no. 3, pp. 91-98, 2007.

[13] Y. Cheng, G. Zhai, and J. Ou, "Direct Time Domain Numerical Analysis of Transient Behavior of a VLFS during Unsteady External Loads in Wave Condition," Abstract and Applied Analysis, vol. 2014, Article ID 628564, 17 pages, 2014. 


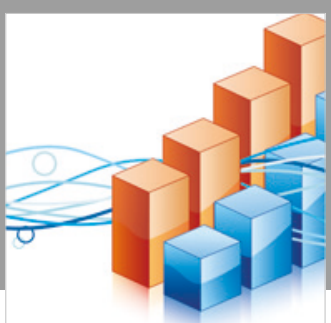

Advances in

Operations Research

\section{-n-m}
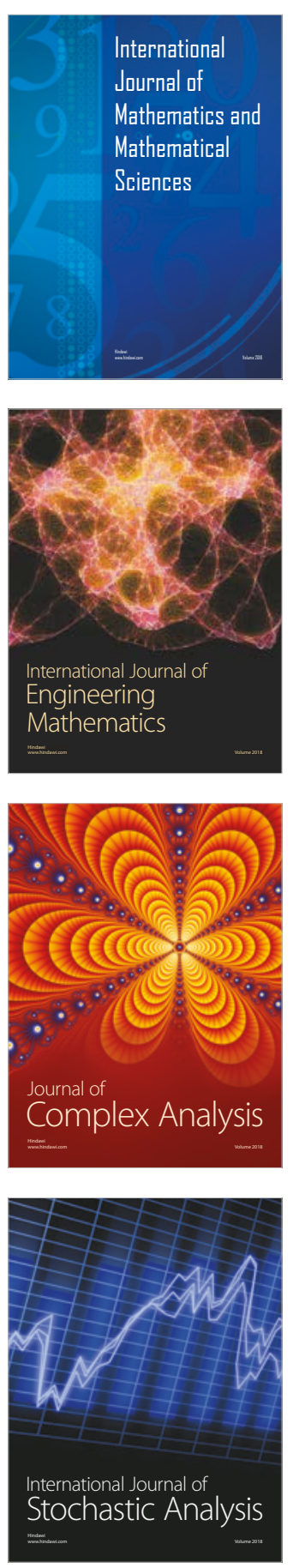
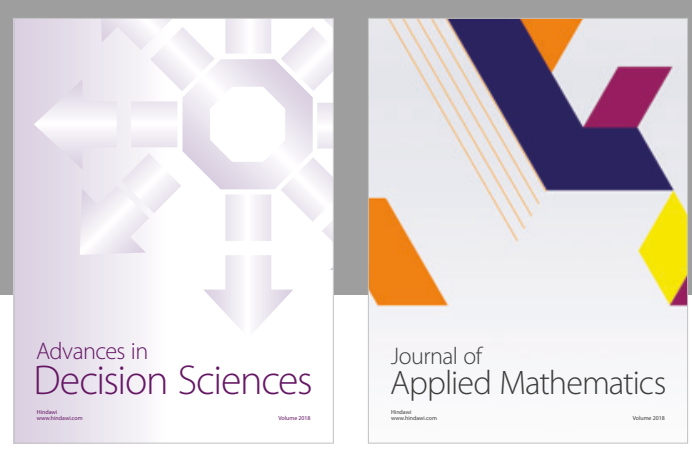

Journal of

Applied Mathematics
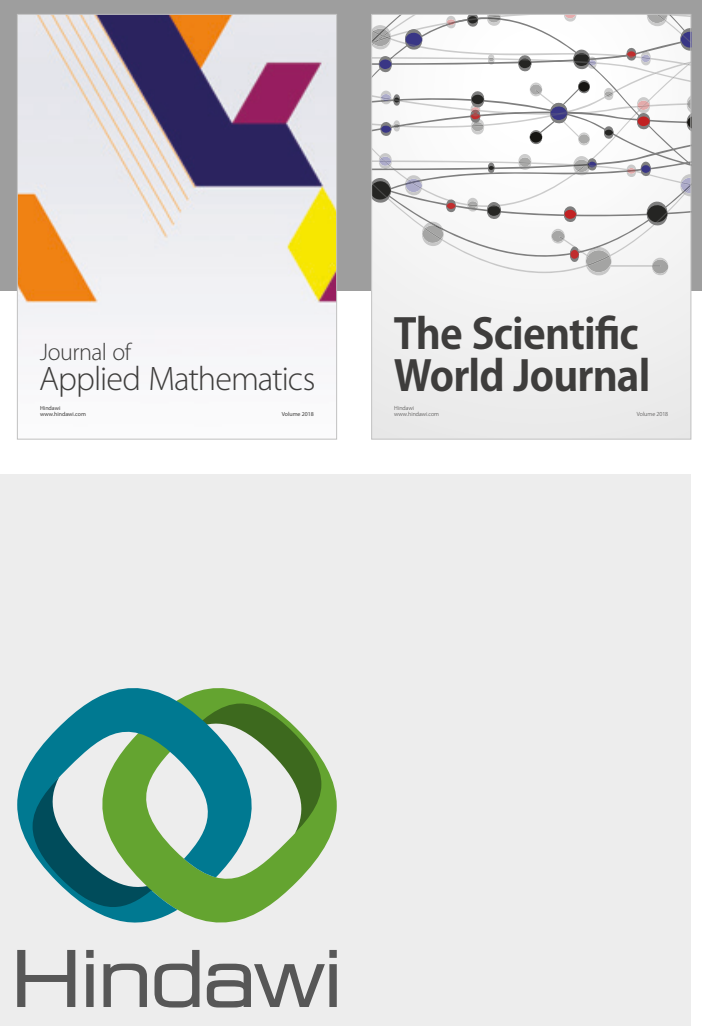

Submit your manuscripts at

www.hindawi.com

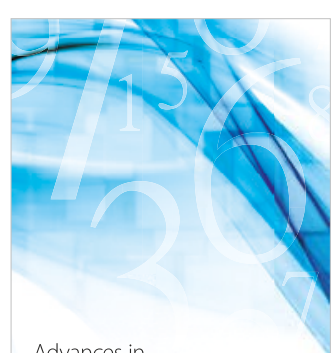

Advances in
Numerical Analysis
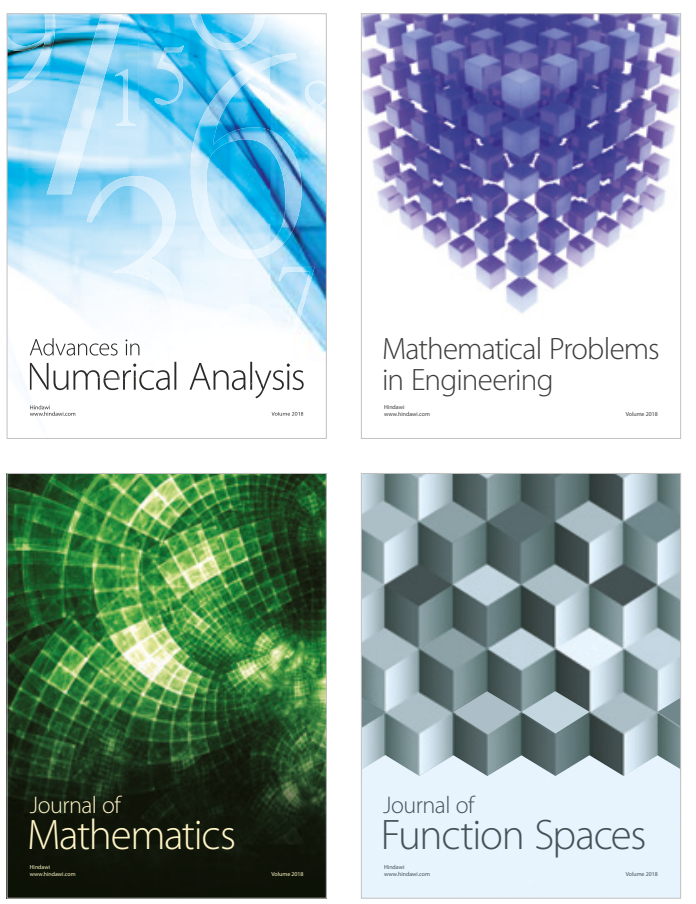

Mathematical Problems in Engineering

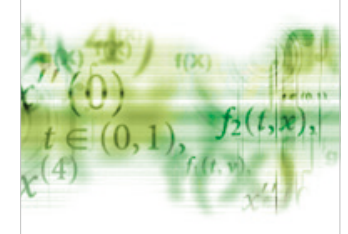

International Journal of

Differential Equations

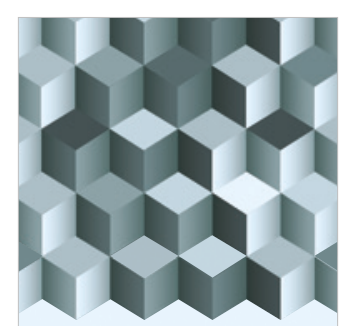

Journal of

Function Spaces

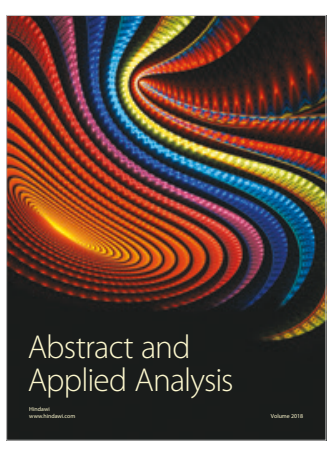

The Scientific

World Journal

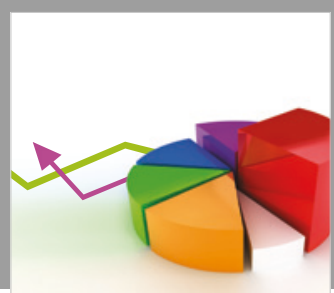

Journal of

Probability and Statistics
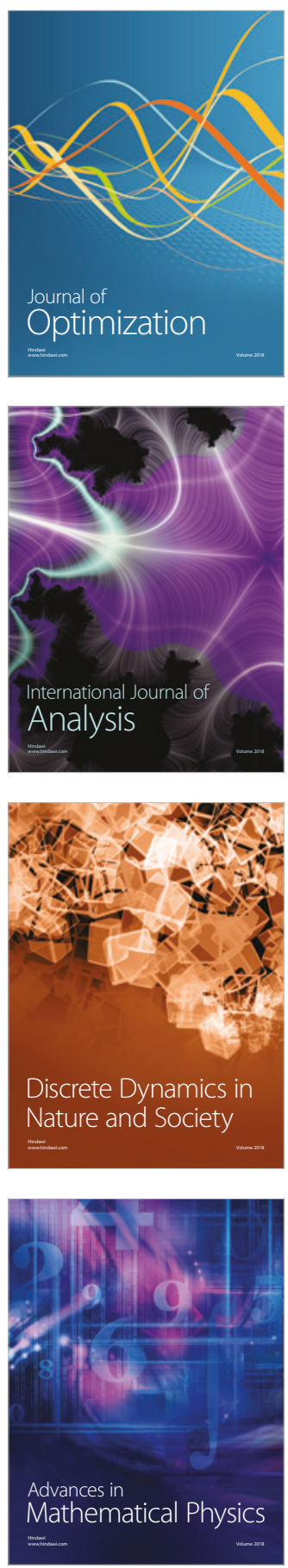